Article

\title{
Signal Consensus in TSP of the Same Grid in Road Network
}

\author{
Dongyuan Li, Chengshuai Li, Zidong Wang, Deqiang Wang, Jianping Xing * and Bo Zhang * \\ School of Microelectronics, Shandong University, Shandong 250100, China; 201511814@mail.sdu.edu.cn (D.L.); \\ 15288840081@163.com (C.L.); 201511840@mail.sdu.edu.cn (Z.W.); wdq_sdu@sdu.edu.cn (D.W.) \\ * Correspondence: xingjp@sdu.edu.cn (J.X.); bobzhangbo@163.com (B.Z.); Tel.: +86-185-5310-1316 (J.X.); \\ +86-151-5314-0108 (B.Z.)
}

Received: 4 September 2017; Accepted: 18 October 2017; Published: 24 October 2017

\begin{abstract}
In this paper, we propose a consensus algorithm with input constraints for traffic light signals in transit signal priority (TSP). TSP ensures control strategy of traffic light signals can be adjusted and applied according to the real-time traffic status, and provides priority for buses. We give the convergence conditions of the consensus algorithms with and without input constraints in TSP respectively and analyze the convergence performance of them by using matrix theory and graph theory, and PTV-VISSIM is used to simulate the traffic accident probability of three cases at intersections. Simulation results are presented that a consensus is asymptotically reached for all weights of priority; the algorithm with input constraints is more suitable for TSP than the algorithm without input constraints, and the traffic accident rate is reduced.
\end{abstract}

Keywords: transit signal priority; signal consensus; road network; input constraints; convergence performance

\section{Introduction}

Transit signal priority (TSP) improves the operational efficiency of transportation and reduces overall delay at an intersection on a per-person basis. The priority of public transport vehicles is higher than that of ordinary social vehicles in TSP [1]. The strategies of TSP provide the signal priority for the priority vehicle by changing the current signal phase length, phase sequence or inserting dedicated phases without affecting the coordination relationship between traffic light signals [2]. The strategies of TSP include passive priority strategy, active priority strategy and real-time strategy [3-6]. Passive priority $[7,8]$ is a strategy of signal timing at an intersection based on historical data without the vehicle detector. Active priority [9-12] means that if a bus is detected close to the intersection, the method of extending, advancing, increasing or jumping phase is adopted to make the bus pass through the intersection smoothly. Through the GPS(Global Positioning System)and AVL(Automatic Vehicle Location) and other advanced information detection devices to estimate the status of the road network and vehicles, real-time strategy $[13,14]$ provides priority signal for buses by optimizing, adjusting the signal timing of the intersection or trunk according to the real-time traffic information. Table 1 shows the main strategies and methods of TSP [15].

The TSP strategies have no significant difference between traffic signal control agents of intersections belonging to the same grid in the road network [16], but their differences will lead to poor driving experiences, and drivers tend to follow the same TSP strategy in the same grid, otherwise it will affect driving behavior and cause confusion. TSP strategies reaching consensus in the same grid play an important role in driving fluency and safety. 
Table 1. The main strategies and methods of Transit signal priority (TSP).

\begin{tabular}{cc}
\hline Strategy & Main Methods \\
\hline Passive priority strategy & Adjustment of cycle length \\
& $\begin{array}{c}\text { Transit movement repetition in the cycle } \\
\text { Green time bias towards transit movement } \\
\text { Phasing design bias towards transit movement } \\
\text { Linking for transit progression }\end{array}$ \\
\hline Active priority strategy & Phase extension \\
& Early phase activation \\
& Special transit phase \\
& Phase suppression \\
\hline Real-time strategy & Delay optimizing \\
& Intersection control \\
& Network control \\
\hline
\end{tabular}

The research of the consensus algorithm is widely used in control, traffic and distributed computation and many fields [17-19]. In 1995, a classical model to simulate the consensus phenomenon of particles was proposed by Vicsek [20] et al., and some interesting results were obtained by simulation. After that, Jadbabaie [21] et al. used the matrix method to analyze the model, and found that the system will eventually reach consensus as long as the network is connected. Reza Olfati-Saber and Murray [22] put forward the theoretical framework of the consensus problem based on the work of Fax and Murray [23,24], designed the most general consensus algorithm, and found the algebraic connectivity of the network can characterize the convergence rate of the system, the condition that the algorithm achieves the average consistency is given and the result was extended to the symmetric consistency algorithm with time delay [25]. In the above study, the status updating of an agent is related to the difference between the neighbor agents, and it will change too fast if the difference is too large, the rapid change of TSP strategy leading to many problems in practice, such as traffic signal confusion and inaccurate timing. We focus on solving this problem in this paper, ensuring that the TSP strategy reaches consensus eventually with a smooth rate to avoid serious traffic problems.

An outline of this paper is as follows: in Section 2, we introduce the basic knowledge of graph theory and matrix theory to better introduce the consensus algorithm, and the consensus algorithm without input constraints and its convergence conditions are demonstrated. In Section 3, we propose a consensus algorithm without input constraints; conditions and proof of its convergence are given, and stability of the algorithm is proved by the Lyapunov function. In Section 4, the application of the consensus algorithm in TSP and the reasons for adopting input constraint are described in detail. In Section 5, the simulation results are presented. Finally, in Section 6, we make our concluding remarks.

\section{Prior Knowledge and Consensus Algorithm}

\subsection{Prior Knowledge}

$\mathbf{G}=\{\mathbf{V}, \mathbf{E}\}$ represents the topology graph and it is composed of set of nodes $\mathbf{V}=\{1,2,3, \ldots, n\}$ and set of edges $\mathbf{E} \in \mathbf{V}^{2}$. There is an edge between node $i$ and node $j$ if they have information interaction. The nodes are disordered in the undirected graph that is $(i, j) \in \mathbf{V} \Leftrightarrow(j, i) \in \mathbf{V}$. The edges have direction in the directed graph.

Suppose $G$ is a directed graph, then: if every vertex is reachable from every other vertex in $G$ then $G$ is a strongly connected graph; the number of head ends adjacent to a vertex is called the in-degree of the vertex and the number of tail ends adjacent to a vertex is its out-degree.

The adjacency matrix $\mathrm{A}$ is used to represent the relationship between the nodes, the values in $\mathrm{A}$ as follows:

$$
a_{i j}=\left\{\begin{array}{l}
1,\left(v_{i}, v_{j}\right) \in E \\
0, \text { otherwise }
\end{array}\right.
$$


There is an edge pointing from node $i$ to node $j$ when $a_{i j}=1$, the degree matrix D represents the value of in-degree of each node in the graph, and the values in $\mathrm{D}$ as follows:

$$
d_{i i}=\left\{\begin{array}{c}
0 ; i \neq j \\
\sum_{j \neq i} a_{i j} .
\end{array}\right.
$$

The Laplacian matrix $\mathbf{L}=\mathbf{D}-\mathbf{A}$ represents the relationship between the nodes and the edges in the graph.

\subsection{Description of Consensus Algorithm}

A topology network $\mathbf{G}=\{\mathbf{V}, \mathbf{E}\}$ is composed of $n$ agents, each node represents an agent $\dot{x}_{i}=u_{i}$. If the state of all agents eventually converges, that is $\left\|x_{i}-x_{j}\right\| \rightarrow 0$, the system tends to be consistent.

The continuous time consensus algorithm of basic fixed topology [26] is described as:

$$
\dot{x}_{i}(t)=\sum_{j \in N_{i}} a_{i j}\left(x_{j}(t)-x_{i}(t)\right) .
$$

Lemma 1. Let $G$ be a connected undirected graph. Then, the algorithm in (3) asymptotically solves an average-consensus problem for all initial states [22].

Lemma 2. Let $G$ be a strongly connected direct graph on $n$ nodes. Then $\operatorname{rank}(\mathbf{L})=n-1$ and all nontrivial eigenvalues of $L$ have positive real parts. Furthermore, suppose $G$ has $c \geq 1$ strongly connected components, then $\operatorname{rank}(\mathbf{L})=n-c$ [27].

Definition 1. A direct graph $G$ is called balanced if $\sum_{j \neq i} a_{i j}=\sum_{j \neq i} a_{j i}$ for all $i \in V$ [26].

Definition 2. A spanning tree $T$ of an undirected graph $G$ is a subgraph that is a tree that includes all of the vertices of $G$, with minimum possible number of edges [28].

Theorem 1. A topology network $\mathbf{G}=\{\mathbf{V}, \mathbf{E}\}$ is composed of $n$ agents. Each agent applies the following consensus algorithm (a). If the network $G$ is a strongly connected direct graph, then:

(i) A consensus is asymptotically reached for all initial states;

(ii) The group decision value is $\alpha=\sum_{i} \omega_{i} x_{i}(0)$ with $\sum_{i} \omega_{i}=1$;

(iii) If the direct graph is balanced, an average-consensus is asymptotically reached and $\alpha=\left(\sum_{i} x_{i}(0)\right) / n$ [22].

\section{Consensus Algorithm with Input Constraints}

\subsection{Description of Consensus Algorithm with Input Constrains}

We proposed a consensus algorithm with input constraints in this paper, it is described as follows:

$$
\begin{aligned}
& \dot{x}_{i}(t)=u_{i}(t), \\
& u_{i}(t)=\sum_{j \in N_{i}} a_{i j} \arctan \left(x_{j}(t)-x_{i}(t)\right) .
\end{aligned}
$$

Obviously, $\operatorname{acr} \tan (x)$ is a bounded function, and $a_{i j}$ is bounded, so $u_{i}(t)$ is bounded. Consensus Algorithm (4) can realize input constraints in the process of information interaction.

Lemma 3. The Laplacian matrix $D$ has at least one zero eigenvalue. The other non-zero eigenvalues are in the left half of the complex plane. In particular, when the graph $G$ has a directed spanning tree, the Laplacian matrix D has only one eigenvalue, which is zero [29]. 
Lemma 4. If the Laplacian matrix $\mathbf{D}$ has only one zero eigenvalue, when $t \rightarrow \infty e^{t \mathbf{L}} \rightarrow \mathbf{1}_{n} \omega^{T}$, where $\omega=\left[\omega_{1}, \ldots, \omega_{n}\right]$ and $\sum_{i=1}^{n} \omega_{i}=1[29]$.

Theorem 2. Consensus Algorithm (4) can gradually form consensus, if and only if the corresponding graph $G$ has a directed spanning tree.

Proof. Sufficiency: When the graph $\mathrm{G}$ has a directed spanning tree, from Lemma 3, the Laplacian matrix $\mathrm{D}$ has only one eigenvalue, which is zero. From Lemma 4 , when $t \rightarrow \infty, x(t)=\mathbf{1}_{n} \omega^{T} x(0)$, we have $x_{i}(t)-x_{j}(t)=0, i, j \in\{1, \ldots, n\}$, consensus Algorithm (4) can gradually form consensus.

Necessity: Suppose consensus Algorithm (4) can gradually form consensus and graph G has no directed spanning tree, then there is no information interaction between at least two agents in this system, they cannot reach consensus, it is the opposite of the hypothesis. Proof finished.

\subsection{Stability Analysis of Consensus Algorithm with Input Constraints}

The stability of the consensus algorithm with input constraints in TSP (4) is proved by using the Lyapunov function.

Proof. Let the Lyapunov function of (4) is

$$
Y=\frac{1}{2} \sum_{i=1}^{n} \sum_{j=1}^{n} a_{i j}\left[\left(x_{i}(t)-x_{j}(t)\right) \arctan \left(x_{i}(t)-x_{j}(t)\right)-\frac{1}{2} \ln \left(1+\left(x_{i}(t)-x_{j}(t)\right)^{2}\right)\right]
$$

Since $x \arctan x-\frac{1}{2} \ln \left(1+x^{2}\right) \geq 0$ and $a_{i j} \geq 0, Y$ is a positive definite function. Its derivation is solved as follows:

$$
\begin{aligned}
& \dot{Y}=\frac{1}{2} \sum_{i=1}^{n} \sum_{j=1}^{n} a_{i j}\left(x_{i}(t)-x_{j}(t)\right)^{\prime} \arctan \left(x_{i}(t)-x_{j}(t)\right) \\
& =\frac{1}{2} \sum_{i=1}^{n} \sum_{j=1}^{n} a_{i j}\left(u_{i}(t)-u_{j}(t)\right)^{T} \arctan \left(x_{i}(t)-x_{j}(t)\right) \\
& =\frac{1}{2} \sum_{i=1}^{n} \sum_{j=1}^{n} a_{i j} u_{i}(t)^{T} \arctan \left(x_{i}(t)-x_{j}(t)\right)-\frac{1}{2} \sum_{i=1}^{n} \sum_{j=1}^{n} a_{i j} u_{j}(t)^{T} \arctan \left(x_{i}(t)-x_{j}(t)\right) \\
& =\frac{1}{2} \sum_{i=1}^{n} \sum_{j=1}^{n} a_{i j} u_{i}(t)^{T} \arctan \left(x_{i}(t)-x_{j}(t)\right)+\frac{1}{2} \sum_{j=1}^{n} \sum_{i=1}^{n} a_{j i} u_{j}(t)^{T} \arctan \left(x_{i}(t)-x_{j}(t)\right)
\end{aligned}
$$

For undirected graph:

$$
a_{i j}=a_{j i}, \dot{Y}=\sum_{i=1}^{n} \sum_{j=1}^{n} a_{i j} u_{i}(t)^{T} \arctan \left(x_{i}(t)-x_{j}(t)\right)=-\sum_{i=1}^{n} u_{i}(t)^{T} u_{i}(t) \leq 0
$$

For directed graph:

$$
\begin{aligned}
& \dot{Y}=\frac{1}{2} \sum_{i=1}^{n} \sum_{j=1}^{n} a_{i j} u_{i}(t)^{T} \arctan \left(x_{i}(t)-x_{j}(t)\right)+\frac{1}{2} \sum_{j=1}^{n} \sum_{i=1}^{n} a_{j i} u_{j}(t)^{T} \arctan \left(x_{i}(t)-x_{j}(t)\right) \\
& =-\frac{1}{2}\left(\sum_{i=1}^{n} u_{i}(t)^{T} u_{i}(t)+\sum_{j=1}^{n} u_{j}(t)^{T} u_{j}(t)\right) \leq 0
\end{aligned}
$$

Above all, the consensus algorithm with input constraints (4) is stable.

\section{Application of Consensus Algorithm with Input Constraints in TSP}

Traffic light signals will be adjusted by TSP strategy according to the real-time traffic condition of intersection when priority vehicles are approaching the intersection. In practice, methods of 
extending the green phase, suppressing the red phase and adjusting the cycle length are widely adopted. The traffic condition of the intersection determines the weight of priority in TSP, the more serious the congestion at the intersection, the greater the weight, so TSP strategy will provide higher priority for the bus. Signal periods of traffic lights in the same grid are the same, and we set the weight of priority at intersection $i$ as the difference between the phase length of green $G_{i}$ and red $R_{i}$ in this paper, that is $P_{i}=G_{i}-R_{i}$.

Road network is divided into grids in the study of intelligent transportation. The traffic light signal agents of the intersections belong to the same grid to carry out communication according to a certain topology network. Figure 1 shows the distribution of intersections in a traffic grid. In practice, when the vehicle passes through different intersections belonging to the same grid, different TSP strategies at different intersections will lead to the influence of driving behavior; drivers tend to follow the same TSP strategy in the same grid, the consensus of TSP strategy in all intersections of the same grid will avoid poor driving experience and confusion. Furthermore, the excessive change in traffic light signals during the information interaction will seriously affect the coordination relationship; compared to the consensus Algorithm (3), the consensus algorithm with input constraints during the information interaction is more applicable for the TSP.

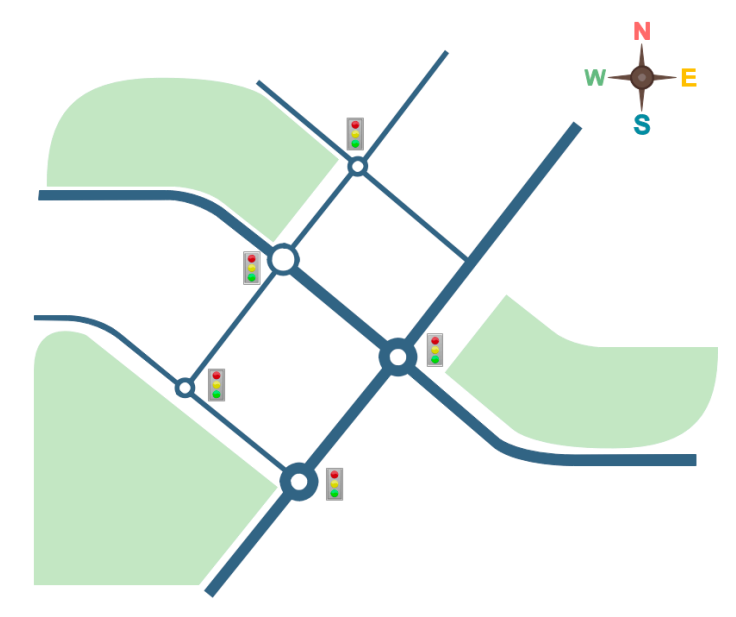

Figure 1. Distribution of intersections in a traffic grid.

\section{Simulation Results}

\subsection{Mathematical Model Establishing}

Combined actual situation with graph theory and matrix theory, the topology network of traffic light signal control agents of the intersections belonging to the same grid is shown in Figure 2, the numbered nodes represent the control agents of traffic lights. The states of the agent represent the weight of priority in TSP, the directed edges represent the relationship of information interaction between the agents, and the corresponding Laplacian matrix in Figure 2 is:

$$
\mathbf{L}=\left(\begin{array}{ccccc}
2 & -1 & 0 & 0 & -1 \\
-1 & 2 & 0 & -1 & 0 \\
0 & -1 & 1 & 0 & 0 \\
-1 & 0 & -1 & 2 & 0 \\
0 & 0 & 0 & -1 & 1
\end{array}\right)
$$

In this paper, we set the initial weight of priority P1 $(0)=15, \mathrm{P} 2(0)=10, \mathrm{P} 3(0)=0, \mathrm{P} 4(5)=0$, and P5 $(0)=8$, respectively. Traffic light signal agents interact information according to the topology network Figure 2 by using consensus algorithm. Eventually the system will converge and be stable, a consensus is asymptotically reached for all weight of priority in the same grid. 


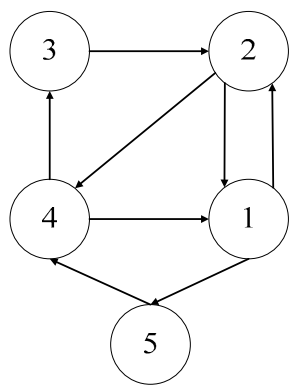

Figure 2. Topology network of intersection traffic lights in a grid.

\subsection{Simulation Results of Consensus Algorithm in TSP}

Figure 3 shows the dynamic processes of 5 weights of priority in Figure 2 by using consensus Algorithm (3), the horizontal axis represents the time and the vertical axis represents the weight of priority. It is can be seen from the simulation results, as time goes on, the system converge and be stable eventually, a consensus is asymptotically reached for all weight of transit signal priority in the same grid.

The state trajectories and the disagreement function $\|\delta\|^{2}$ associated with consensus Algorithm (3) are shown in Figure 4. It can reflect the rate of convergence of the consensus algorithm.

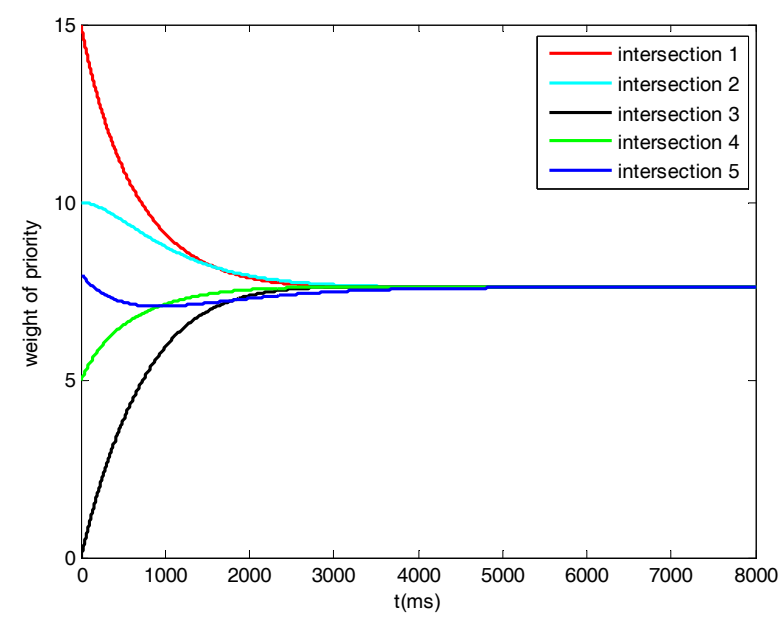

Figure 3. Simulation results of consensus algorithm in transit signal priority (TSP).

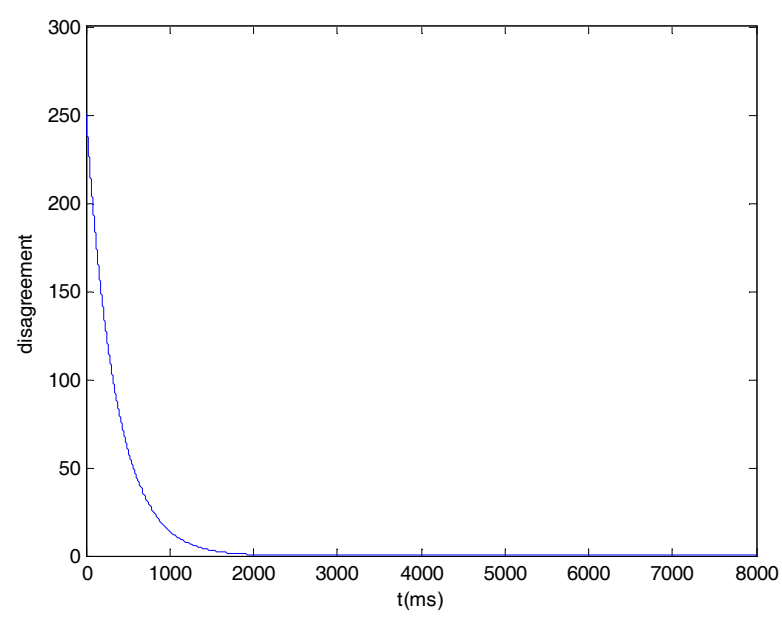

Figure 4. State trajectories and the disagreement function $\|\delta\|^{2}$. 


\subsection{Simulation Results of Consensus Algorithm with Input Constraints in TSP}

Figure 5 shows the dynamic processes of 5 weights of priority in Figure 2 by using consensus Algorithm (4), the state trajectories and the disagreement function $\|\delta\|^{2}$ associated with consensus Algorithm (4) are shown in Figure 6.

It is can be seen from the simulation results that the consensus is also reached for all weight of transit signal priority in the same grid by using consensus Algorithm (4).

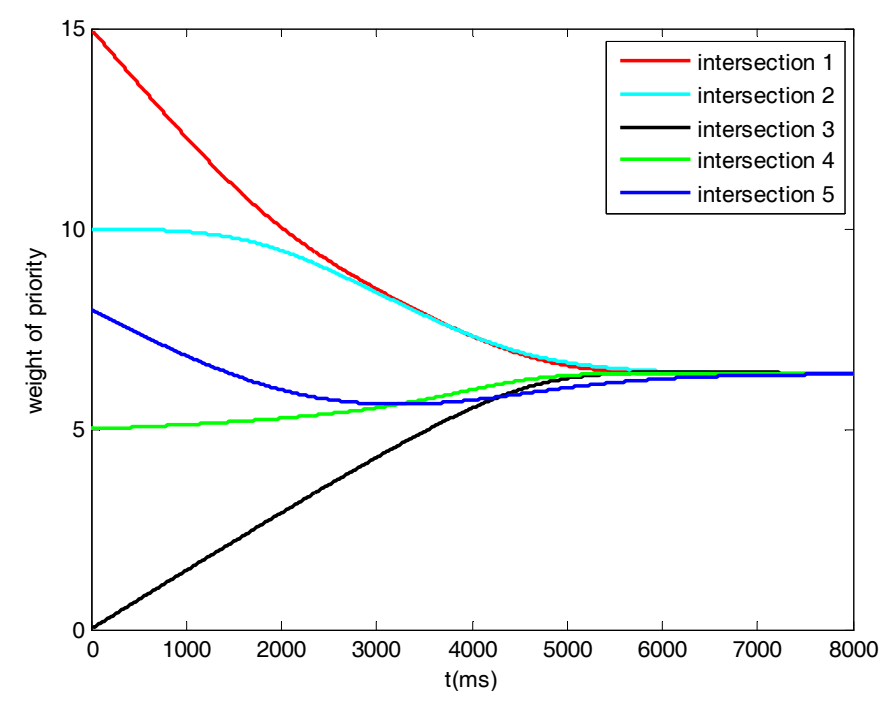

Figure 5. Simulation results of consensus algorithm with input constraints in TSP.

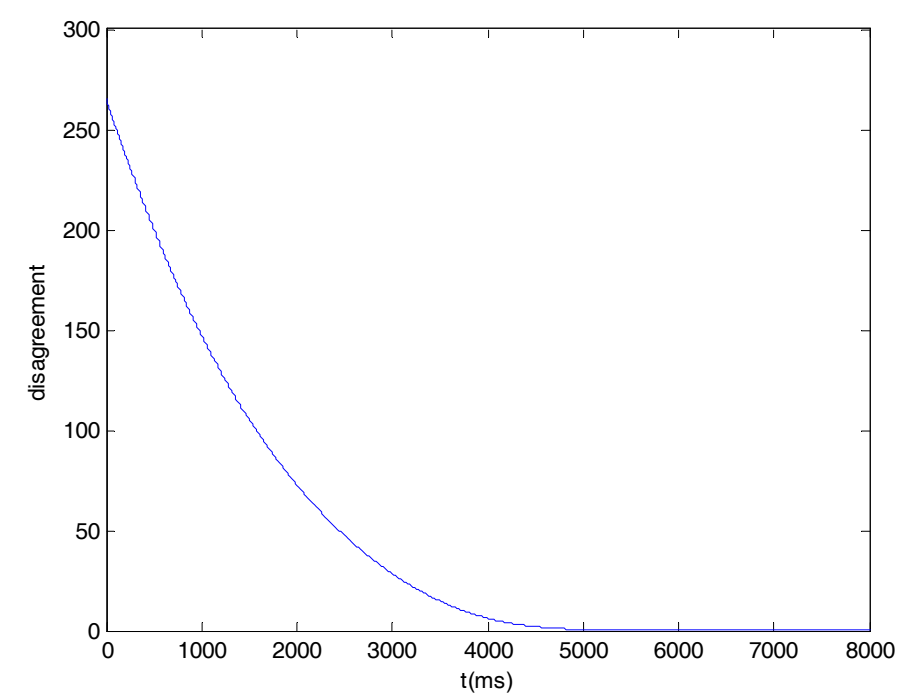

Figure 6. State trajectories and the disagreement function $\|\delta\|^{2}$.

Compared Figure 6 with Figure 4, the rate of convergence of the consensus Algorithm (4) is slower and smoother than (3). Information interaction between the nodes is limited and more stable in consensus Algorithm (4), it is more suitable for the signal consensus in the TSP.

\subsection{Simulation Results of Probability of Traffic Accidents at Intersections}

The above simulation results show the comparison of the convergence rates between the consensus algorithms with and without input constraints. This section will prove that the TSP strategies reaching consensus eventually with a smooth rate can avoid serious traffic accidents through simulation 
experiments at intersections. The traffic accidents are largely caused by the excessive acceleration of the drivers. According to statistics, most rear-end accidents are caused by the emergency braking of vehicles at the intersection. The different TSP strategies in the same grid will lead to poor driving experiences, and untimely responses to the change in signal strategy can cause the driver to brake too quickly. Traffic simulation tool VISSIM is used to simulate the traffic accident probability of 5 intersections in Figure 2. Three cases are analyzed and compared: TSP without consensus algorithm, TSP with consensus algorithm (3) and TSP with consensus algorithm (4). The same settings for the three simulations are as follows: we assume that when the road load exceeds $50 \%$, there will be a $10 \%$ probability of traffic accidents if the acceleration of the vehicle braking is greater than $5 \mathrm{~m} / \mathrm{s}^{2}$. The VISSIM simulation interface is shown in Figure 7, we simulated the traffic flow of 5 intersections for a month, and the number of traffic accidents as shown in Table 2.

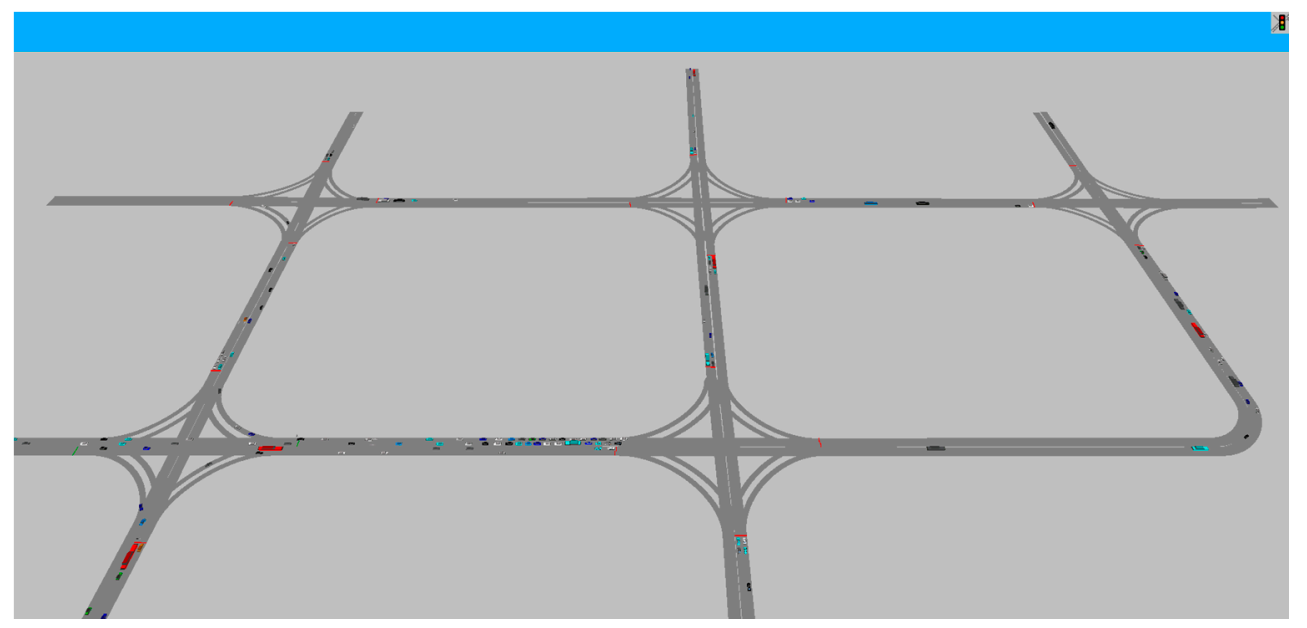

Figure 7. PTV-VISSIM (v4.3, PTV Group, Karlsruhe, Germany) simulation interface.

Table 2. Number of traffic accidents of 3 cases at intersections.

\begin{tabular}{cccc}
\hline $\begin{array}{c}\text { Intersection } \\
\text { Number }\end{array}$ & $\begin{array}{c}\text { TSP without Consensus } \\
\text { Algorithm }\end{array}$ & $\begin{array}{c}\text { TSP with Consensus } \\
\text { Algorithm (3) }\end{array}$ & $\begin{array}{c}\text { TSP with Consensus } \\
\text { Algorithm (4) }\end{array}$ \\
\hline Intersection 1 & 5 & 3 & 2 \\
Intersection 2 & 3 & 2 & 2 \\
Intersection 3 & 4 & 2 & 1 \\
Intersection 4 & 4 & 3 & 3 \\
Intersection 5 & 3 & 2 & 2 \\
\hline
\end{tabular}

Simulation results show that, compared with the former two cases, the proposed algorithm with input constraints in TSP reduces the number of traffic accidents in the same grid, and plays an effective role in TSP.

\section{Summary}

In this paper, we focus on the TSP strategy consensus problem of the same grid in road networks. Consensus algorithms with and without input constraints are described by using graph theory and matrix theory, and their convergence conditions are proved. The stability of the consensus algorithm with input constraints, which we propose in this paper, is proved by the Lyapunov function. The two consensus algorithms are simulated and analyzed, the simulation results show that: on the premise that both can reach consensus, the algorithm with input constraints converges slower than algorithms without input constraints; the weight of priority varies more gently, it is more suitable for 
TSP in practice. In the end, simulation results of traffic accident probability of 3 cases at intersections are given, and the proposed algorithm can decrease traffic accident rates effectively.

Acknowledgments: This work is supported by China Postdoctoral Science Foundation funded project (2016M601152), the National Natural Science Foundation of China under grant 61603215 and Shandong Province independent innovation major project (Beidou high-precision positioning equipment industrialization, network service platform).

Author Contributions: Dongyuan Li, Chengshuai Li and Jianping Xing conceived and designed the study. Dongyuan Li, Zidong Wang, Deqiang Wang, and Bo Zhang performed the experiments. Jianping Xing provided the data. Dongyuan Li and Bo Zhang analyzed the data. Dongyuan Li and Chengshuai Li proof the algorithm, Dongyuan Li wrote the paper. All authors read and approved the paper.

Conflicts of Interest: The authors declare no conflict of interest.

\section{References}

1. ITS America. Transit Signal Priority (TSP): A Planning and Implementation Handbook; United States Department of Transportation: Washington, DC, USA, 2005.

2. Bertini, R.L.; Glasgow, C.; Peters, J. An Overview of Transit Signal Priority; ITS America: Washington, DC, USA, 2004.

3. Transit Cooperative Research Program. Improved Traffic Light Signal Priority for Transit; TCRP Project A-16; Transportation Research Board, National Research Council: Washington, DC, USA, 1998.

4. Sunkari, S.R.; Beasley, P.S.; Urbanik, T., II; Fambro, D.B. Model to evaluate the impacts of bus priority on signalized intersections. Transp. Res. Rec. 1995, 117-123.

5. Daniel, J.; Lieberman, E.; Srinivasan, R. Assess Impacts and Benefits of Traffic Light Signal Priority for Busses; FHWA-NJ-2004-013; FHWA: Washington, DC, USA, 2004.

6. Gang-Len, C.; Vasudevan, M.; Chih-Chiang, S.U. Bus-Preemption under Adaptive Signal Control Environments. Transp. Res. Board 1995, 146-154. Available online: https://www.researchgate. net/publication/288884528_Bus-preemption_under_adaptive_signal_control_environments (accessed on 23 October 2017).

7. Yagar, S. Efficient Transit Priority at Intersections. Transp. Res. Rec. 1993, 10-15. Available online: http: / / onlinepubs.trb.org/Onlinepubs/trr/1993/1390/1390-002.pdf (accessed on 23 October 2017).

8. Skabardonis, A. Control Strategies for Transit Priority; TRB, National Research Council: Washington, DC, USA, 2000.

9. Rakha, H.; Zhang, Y. Sensitivity Analysis of Transit Signal Priority Impacts on Operation of a Signalized Intersection. J. Transp. Eng. 2004, 130, 796-804. [CrossRef]

10. Roark, J.J. Synthesis of Transit Practice-Enforcement of Priority Treatment for Buses on Urban Streets; National Cooperative Transit Research \& Development Program, TRB, National Research Council: Washington, DC, USA, 1982.

11. Baker, R.J.; Collura, J.; Dale, J.J.; Head, L.; Hemily, B.; Ivanovic, M.; Jarzab, J.T.; McCormick, D.; Obenberger, J.; Smith, L.; et al. An Overview of Transit Signal Priority. Computers 2002. Available online: http:/ /xueshu.baidu.com/s?wd=paperuri:(315181fa6b80d25347091b16c6441f26)\&filter=sc_long_ sign\&tn=SE_xueshusource_2kduw22v\&sc_vurl=http:/ / trid.trb.org / view /723986\&ie=utf-8\&sc_us= 13110307943409424370 (accessed on 23 October 2017).

12. Heydecker, B.G. Capacity at a Signal-controlled Junction Where There Is Priority for Buses. Transp. Res. 1983, 17, 341-357. [CrossRef]

13. Lee, J.; Shalaby, A.; Greenough, J.; Bowie, M.; Hung, S. Advanced Transit Signal Priority Control with Online Microsimulation-Based Transit Prediction Model. Transp. Res. Rec. J. Transp. Res. Board 2005, 1925, 185-194. [CrossRef]

14. Yagar, S.; Han, B. A Procedure for Real-Time Signal Control that Considers. Transit Interference. Transp. Res. 1994, 28, 315-331.

15. Ma, W.; Yang, X. Study on priority control strategy of bus signal. J. Urban Transp. 2010, 8, 70-78.

16. Jenelius, E.; Mattsson, L.G. Road network vulnerability analysis of area-covering disruptions: A grid-based approach with case study. Transp. Res. Part A 2012, 46, 746-760. [CrossRef] 
17. Savkin, A.V. Coordinated collective motion of groups of autonomous mobile robots: Analysis of Vicsek's model. IEEE Trans. Autom. Control 2004, 49, 981-982. [CrossRef]

18. Gazi, V.; Passino, K.M. Stability analysis ofswarms. IEEE Trans. Autom. Control 2003, 48, 692-697. [CrossRef]

19. Liu, Y.; Passino, K.M.; Polycarpou, M.M. Stability analysis of M-dimensional asynchronous swarms with a fixed communication topology. IEEE Trans. Autom. Control 2003, 48, 76-95.

20. Vicsek, T.; Cziroo'k, A.; Ben-Jacob, E.; Cohen, I.; Shochet, O. Novel type of phase transition in a system of self-deriven particles. Phys. Rev. Lett. 1995, 75, 1226-1229. [CrossRef] [PubMed]

21. Jadbabaie, A.; Lin, J.; Morse, A.S. Coordination of groups of mobile autonomous agents using nearest neighbor rules. IEEE Trans. Autom. Control 2003, 48, 988-1001. [CrossRef]

22. Olfati-Saber, R.; Murray, R.M. Consensus problems in networks of agents with switching topology and time-delays. IEEE Trans. Autom. Control 2004, 49, L520-L533. [CrossRef]

23. FaxJ, A. Optimal and Cooperative Control of Vehicle Formations. Ph.D. Thesis, Control Dynamical System, California Institute of Technology, Pasadena, CA, USA, 2001.

24. FaxJ, A.; Murray, R.M. Information flow and cooperative control of vehicle formations. IEEE Trans. Autom. Control 2004, 49, 1465-1476.

25. Wen, Y. Study on Consistency of Multi-Agent System. Ph.D. Thesis, Shanghai Jiaotong University, Shanghai, China, 2009.

26. Olfati-Saber, R.; Fax, J.A.; Murray, R.M. Consensus and Cooperation in Networked Multi-Agent Systems. Proc. IEEE 2007, 95, 215-233. [CrossRef]

27. Saber, R.O.; Murray, R.M. Agreement problems in networks with directed graphs and switching topology. In Proceedings of the IEEE Conference on Decision and Control, Maui, HI, USA, 9-12 December 2003; Volume 4, pp. 4126-4132.

28. Gallager, R.G.; Humblet, P.A.; Spira, P.M. A Distributed Algorithm for Minimum-Weight Spanning Trees; ACM: New York, NY, USA, 1983.

29. Lei, B. Research on Cooperative Control Problem of Swarm Robots System. Ph.D. Thesis, Wuhan University of Technology, Wuhan, China, 2009.

(C) 2017 by the authors. Licensee MDPI, Basel, Switzerland. This article is an open access article distributed under the terms and conditions of the Creative Commons Attribution (CC BY) license (http:/ / creativecommons.org/licenses/by/4.0/). 